\title{
Pseudoaneurisma: Rara Complicação do Acesso Radial
}

\author{
Luciano Nunes dos Santos ${ }^{1}$, Rodrigo B. Esper ${ }^{1}$, Luiz Fernando Ybarra' ${ }^{1}$, Henrique B. Ribeiro', \\ Carlos A. Campos ${ }^{1}$, Augusto C. Lopes Jr. ${ }^{1}$, Expedito E. Ribeiro'
}

\section{RESUMO}

A via radial apresenta menores taxas de complicações vasculares para realização de cinecoronariografia. O pseudoaneurisma da artéria radial é uma complicação rara desse procedimento. O diagnóstico precoce é essencial para evitar complicações como ruptura espontânea, isquemia e síndrome compartimental. Neste relato é descrito um caso de pseudoaneurisma da artéria radial direita após realização de cinecoronariografia eletiva. O diagnóstico foi confirmado por ultrassonografia com Doppler, que evidenciou pseudoaneurisma da artéria radial na face anteromedial do antebraço, medindo $3,7 \times 2,5 \mathrm{~cm}$, com colo de $0,7 \mathrm{~cm}$. O tratamento foi feito com curativo compressivo local e nova ultrassonografia com Doppler, realizada 12 horas após, demonstrou resolução do pseudoaneurisma.

DESCRITORES: Falso aneurisma. Artéria radial. Angiografia coronária.

A utilização da via radial para realização de cinecoronariografia foi proposta inicialmente por Campeau', em 1989, sendo logo incorporada à prática intervencionista. Essa técnica apresenta vantagens como a possibilidade de deambulação precoce, menores taxas de complicações vasculares e menor custo hospitalar ${ }^{2}$, sendo ainda uma alternativa em pacientes com doença vascular periférica obstrutiva ilíacofemoral. O pseudoaneurisma da artéria radial é uma complicação rara associada a esse procedimento e mais frequentemente localizado na região do acesso vascular. Os métodos atuais de tratamento para o pseudoaneurisma da artéria radial são semelhantes àqueles realizados nos pseudoaneurismas da artéria femoral,

${ }^{1}$ Instituto do Coração do Hospital das Clínicas da Faculdade de Medicina da Universidade de São Paulo (InCor/HCFMUSP) - São Paulo, SP, Brasil. Correspondência: Luciano Nunes dos Santos. Rua Alves Guimarães, 642 - Pinheiros - São Paulo, SP, Brasil - CEP 05410-001 E-mail: santos.In@uol.com.br

Recebido em: 6/6/2011 • Aceito em: 30/8/2011
ABSTRACT

\section{Pseudoaneurysm: A Rare Complication of Radial Access}

The radial approach has lower vascular complication rates for coronary angiography. Radial artery pseudoaneurysm is a rare complication of this procedure. Early diagnosis is essential to avoid complications such as spontaneous rupture, ischemia and compartment syndrome. We describe a case of pseudoaneurysm of the right radial artery after elective coronary angiography. Diagnosis was confirmed by Doppler ultrasound, which revealed a pseudoaneurysm of the radial artery on the anteromedial surface of the forearm, measuring $3.7 \times 2.5 \mathrm{~cm}$, with a neck of $0.7 \mathrm{~cm}$. Treatment was done with local compressive dressing and a new Doppler ultrasound, performed 12 hours later, demonstrated resolution of the pseudoaneurysm.

KEY-WORDS: Aneurysm, false. Radial artery. Coronary angiography.

incluindo compressão mecânica manual ou guiada por ultrassom, injeção de trombina percutânea ${ }^{3}$ e cirurgia convencional. ${ }^{4}$

\section{RELATO DO CASO}

Paciente do sexo masculino, com 64 anos de idade, ex-tabagista e hipertenso, com antecedente de aneurisma de aorta abdominal e angioplastia da artéria descendente anterior com implante de stent não-farmacológico há seis anos. Apresentava angina estável aos esforços habituais e estava em uso de ácido acetilsalicílico, sendo encaminhado para realização de cinecoronariografia eletiva, que evidenciou oclusão intrastent da artéria descendente anterior. O procedimento foi realizado pela via radial direita, utilizando introdutor 6 F, 5.000 unidades de heparina não-fracionada e $200 \mu \mathrm{g}$ de nitroglicerina. Ao final do exame, foi realizada hemostasia por meio de curativo compressivo com gaze e esparadrapo no local da punção, permanecendo em observação hospitalar por três horas, sem intercorrências antes da alta hospitalar. Cerca de cinco horas depois, o paciente retornou ao pronto-so- 
corro com queixa de dor e grande aumento de volume no local da punção arterial, sendo observado extenso hematoma envolvendo a face anterior do antebraço (Figura 1). Pulso radial estava presente e a perfusão periférica estava adequada. A ultrassonografia com Doppler do membro superior direito evidenciou pseudoaneurisma na face anteromedial do antebraço, partindo da artéria radial, medindo $3,7 \times 2,5 \mathrm{~cm}$, com colo de 0,7 cm, associado a importante edema dos planos cutâneo e muscular adjacentes (Figura 2). Optou-se por realizar curativo compressivo no local da punção arterial, para tentativa de oclusão mecânica do pseudoaneurisma. Após 12 horas, foi realizado novo ultrassom com Doppler, que não detectou fluxo em meio à loja da coleção, confirmando o fechamento do colo do pseudoaneurisma (Figura 3). As artérias radial e ulnar apresentavam trajeto e calibre preservados, com fluxo trifásico compatível com a normalidade. O hematoma na face anterior do antebraço media $6,4 \times 3,9 \times 2 \mathrm{~cm}$. Durante a interna-

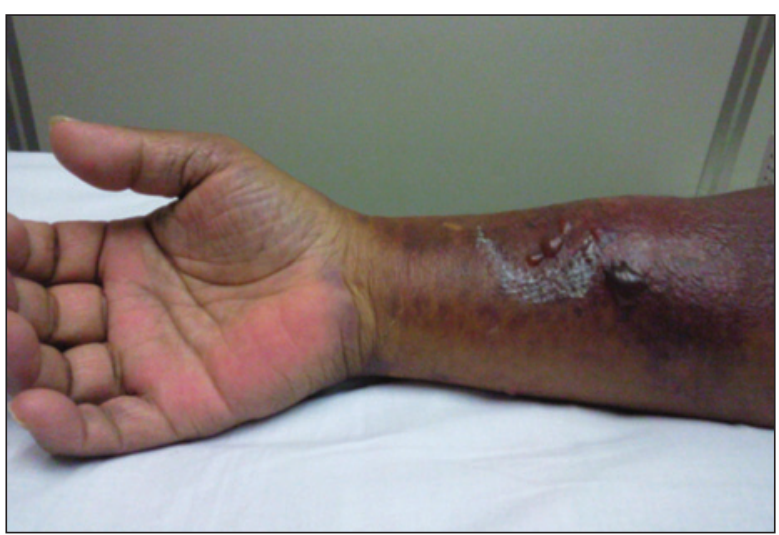

Figura 1 - Imagem do antebraço direito, evidenciando grande hematoma próximo ao local da punção da artéria radial.

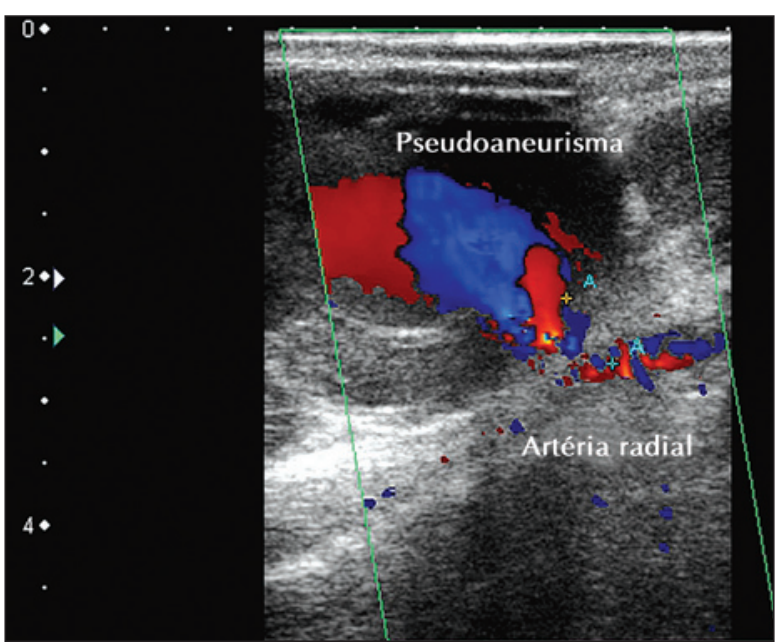

Figura 2 - Ultrassonografia com Doppler em cores (corte longitudinal do antebraço direito), demonstrando fluxo laminar pelo colo do pseudoaneurisma da artéria radial direita. ção o paciente apresentou melhora da dor e do edema, mantendo-se afebril, sem sinais de infecção ou de síndrome compartimental. Recebeu alta após três dias, retornando em um mês para consulta ambulatorial, mantendo bom pulso radial e adequada perfusão do membro superior direito, com regressão do hematoma local.

\section{DISCUSSÃo}

O acesso radial para realização de procedimentos coronários tem apresentado crescente utilização nas últimas décadas, com menores taxas globais de complicações e menor custo hospitalar² em comparação com a abordagem femoral. De acordo com uma metanálise, o acesso radial apresenta risco $73 \%$ menor de sangramento maior, quando comparado com a via femoral, ocorrendo, respectivamente, em $0,05 \%$ e $2,3 \% .^{5}$ Nesse mesmo estudo houve tendência a redução do desfecho composto de morte, infarto ou acidente vascular cerebral $[2,5 \%$ vs. $3,8 \%$, respectivamente; odds ratio (OR) 0,71 ; intervalo de confiança de 95\% (IC 95\%) 0,49-1,01], além de redução estatisticamente significante do tempo de internação hospitalar. Mais recentemente, o estudo multicêntrico Radial Versus Femoral Access for Coronary Angiography and Intervention in Patients with Acute Coronary Syndromes (RIVAL) ${ }^{6}$ randomizou mais de 7 mil pacientes admitidos por síndrome coronária aguda, demonstrando que a via radial levou à redução da incidência de complicações vasculares maiores em relação ao acesso femoral $[1,4 \%$ vs. 3,7\%; hazard ratio (HR) 0,37; IC 95\% 0,27-0,52; $\mathrm{P}<0,001]$.

Uma complicação rara da cinecoronariografia pelo acesso radial é a formação do pseudoaneurisma no local da punção, com incidência estimada entre 0,029\% e $0,19 \%$. $^{6-9}$ A formação do pseudoaneurisma ocorre por ruptura e lesão da parede arterial no local da canulação radial, podendo levar a hemorragia e hematoma local.

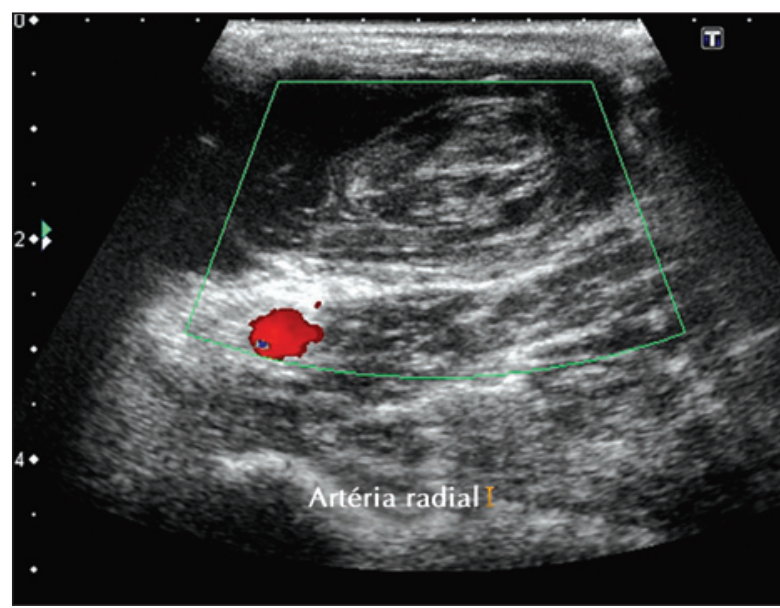

Figura 3 - Imagem com Doppler em cores (corte axial do antebraço direito), evidenciando ausência de fluxo em meio à loja da coleção e inferindo oclusão do colo do pseudoaneurisma. Observa-se ainda fluxo da artéria radial íntegro e extenso hematoma na face anterior do antebraço. 
Essas lesões vasculares estão associadas a múltiplas tentativas de punção, uso de anticoagulantes, utilização de introdutores de grande calibre e infecção. ${ }^{10} \mathrm{O}$ desenvolvimento do pseudoaneurisma tem evolução clínica variável, podendo ocorrer algumas horas após a realização do exame ou ser observado dias (7-40 dias) depois do procedimento.

O diagnóstico e o tratamento precoces do pseudoaneurisma são essenciais para manter a adequada perfusão da mão e minimizar as complicações, como ruptura espontânea, isquemia da mão (especialmente se a artéria radial é dominante) e síndrome compartimental. Medidas preventivas devem ser utilizadas para reduzir a ocorrência de pseudoaneurisma e outras complicações vasculares, incluindo a preparação estéril do procedimento, evitar múltiplas punções no mesmo local, uso de técnica adequada e manipulação correta do equipamento endovascular. ${ }^{11}$

O manejo tradicional do pseudoaneurisma inclui compressão mecânica manual ou guiada por ultrassom, injeção percutânea de trombina ou cirurgia. A compressão guiada por ultrassom possui ampla aceitação, em decorrência de sua segurança e eficácia. A compressão direta do colo pelo transdutor causa estase sanguínea, formação de coágulo local e oclusão do pseudoaneurisma. A injeção de trombina percutânea com auxílio da ultrassonografia com Doppler no pseudoaneurisma da artéria femoral apresenta taxa de sucesso entre 93\% e 100\%. Contudo, existem apenas poucos relatos descrevendo esse tratamento em pseudoaneurisma de pequenas artérias periféricas. Essa técnica pode se associar com risco de embolização de trombina para a circulação distal, ocasionando trombose vascular e isquemia periférica. A manobra combinada de compressão do pseudoaneurisma e liberação no momento da injeção de trombina pode aumentar a margem de segurança. ${ }^{12}$

A excisão cirúrgica do pseudoaneurisma ou a ligadura da artéria radial podem ser realizadas com segurança, como tem sido demonstrado em estudos sobre as lacerações das artérias do punho e na retirada da artéria radial para utilização como enxerto na cirurgia de revascularização miocárdica. ${ }^{13,14} \mathrm{~A}$ reconstrução vascular deve ser reservada para pseudoaneurisma complicado ou roto, falha do tratamento conservador ou isquemia crítica da mão com chance de perda do membro.

Neste caso relatado, realizou-se curativo compressivo no local do pseudoaneurisma do antebraço direito. À ultrassonografia com Doppler de controle, realizada após 12 horas, foi demonstrada resolução do pseudoaneurisma. A localização radial favorece a realização de curativo compressivo e o ultrassom com Doppler pode facilitar a identificação precisa do local a ser comprimido.

O acesso radial é considerado alternativa segura e eficaz em relação à via femoral, sendo a incidência de complicações relacionadas ao sítio de punção significantemente menor. Relatamos o primeiro caso de pseudoaneurisma de artéria radial após cinecoronariografia descrito na literatura brasileira. O tratamento foi realizado por curativo compressivo, sendo possivelmente uma alternativa eficaz aos outros métodos de oclusão do pseudoaneurisma radial.

\section{CONFLITO DE INTERESSES}

Os autores declaram não haver conflito de interesses relacionado a este manuscrito.

\section{REFERÊNCIAS}

1. Campeau L. Percutaneous radial artery approach for coronary angiography. Cathet Cardiovasc Diagn. 1989;16(1):3-7.

2. Cooper CJ, El-Shiekh RA, Cohen DJ, Blaesing L, Burket MW, Basu A, et al. Effect of transradial access on quality of life and cost of cardiac catheterization: a randomized comparison. Am Heart J. 1999;138(3 Pt 1):430-6.

3. Ferguson JD, Whatling PJ, Martin V, Walton J, Banning AP. Ultrasound guided percutaneous thrombin injection of iatrogenic femoral artery pseudoaneurysms after coronary angiography and intervention. Heart. 2001;85(4):E5.

4. Morgan R, Belli AM. Current treatment methods for postcatheterization pseudoaneurysms. J Vasc Interv Radiol. 2003; 14(6):697-710

5. Agostoni $\mathrm{P}$, Biondi-Zoccai GG, de Benedictis $\mathrm{ML}$, Rigattieri S, Turri M, Anselmi M, et al. Radial versus femoral approach for percutaneous coronary diagnostic and interventional procedures; systematic overview and meta-analysis of randomized trials. J Am Coll Cardiol. 2004;44(2):349-56.

6. Jolly SS, Yusuf S, Cairns J, Niemelä K, Xavier D, Widimsky P, et al. Radial versus femoral access for coronary angiography and intervention in patients with acute coronary syndromes (RIVAL): a randomised, parallel group, multicentre trial. Lancet. 2011;377(9775):1409-20.

7. Falk PS, Scuderi PE, Sherertz RJ, Motsinger SM. Infected radial artery pseudoaneurysms occurring after percutaneous cannulation. Chest. 1992;101(2):490-5.

8. Komorowska-Timek E, Teruya TH, Abou-Zamzam AM Jr, Papa D, Ballard JL. Treatment of radial and ulnar artery pseudoaneurysms using percutaneous thrombin injection. J Hand Surg Am. 2004;29(5):936-42.

9. Sanmartin M, Cuevas D, Goicolea J, Ruiz-Salmerón R, Gómez $M$, Argibay $V$. Vascular complications associated with radial artery access for cardiac catheterization. Rev Esp Cardiol. 2004;57(6):581-4.

10. Kang SS, Labropoulos N, Mansour MA, Michelini M, Filliung D, Baubly MP, et al. Expanded indications for ultrasoundguided thrombin injection of pseudoaneurysms. J Vasc Surg. 2000;31(2):289-98.

11. Pancholy S, Coppola J, Patel T, Roke-Thomas M. Prevention of radial artery occlusion-patent hemostasis evaluation trial (PROPHET study): a randomized comparison of traditional versus patency documented hemostasis after transradial catheterization. Catheter Cardiovasc Interv. 2008;72(3):335-40.

12. Pozniak MA, Mitchell C, Ledwidge M. Radial artery pseudoaneurysm: a maneuver to decrease the risk of thrombin therapy. J Ultrasound Med. 2005;24(1):119-22.

13. Johnson M, Ford M, Johansen K. Radial or ulnar artery laceration. Repair or ligate? Arch Surg. 1993;128(9):971-4; discussion 974-5.

14. Starnes SL, Wolk SW, Lampman RM, Shanley CJ, Prager RL, Kong BK, et al. Noninvasive evaluation of hand circulation before radial artery harvest for coronary artery bypass grafting. J Thorac Cardiovasc Surg. 1999;117(2):261-6. 